\title{
Effectiveness of septoplasty on pulmonary function tests in symptomatic deviated nasal septum cases: a prospective study
}

\author{
Vidya Bhargavan Panicker, B.P. Belaldavar*
}

\begin{abstract}
Department of Otorhinolaryngology and Head and Neck Surgery, Jawaharlal Nehru Medical College, Belgaum, Karnataka, India
\end{abstract}

Received: 08 March 2018

Accepted: 05 April 2018

\section{*Correspondence:}

Dr. B.P. Belaldavar,

E-mail: puttawwa@rediffmail.com

Copyright: (C) the author(s), publisher and licensee Medip Academy. This is an open-access article distributed under the terms of the Creative Commons Attribution Non-Commercial License, which permits unrestricted non-commercial use, distribution, and reproduction in any medium, provided the original work is properly cited.

\section{ABSTRACT}

Background: Deviation of normal pulmonary functions leads to dysfunction of the respiratory system and this affects the functions and vitality of other related systems. Pulmonary function tests give valuable information on the state of airways, lung volumes and lung function. Hence, the present study aimed to evaluate the effectiveness of septoplasty on pulmonary function tests in symptomatic deviated nasal septum cases.

Methods: A total of 35 patients (septoplasty: 31; septorhinoplasty: 4) with deviated nasal septum were involved in the study. Demographic data, clinical and physical examination including anterior and posterior rhinoscopy was performed. RMS Helios 702 spirometer was used to perform pre- and postoperative pulmonary function tests. Forced vital capacity (FVC), forced expiratory volume (FEV1) in 1 second, peak expiratory flow rate, and the ratio of FEV1 to FVC were the parameters measured. SPSSV. 17 was used to analyse the data.

Results: The deviation was most commonly seen on the left side (54\%), affecting mostly the cartilaginous septum $(54 \%)$. Among patients with septoplasty, the postoperative values of FVC $(p<0.05)$, FEV1 $(p<0.05)$, and peak expiratory flow $(\mathrm{p}<0.05)$ were higher than the preoperative values and the results were statistically significant. Age, gender, laterality and duration of deviation, headache and inferior turbinate hypertrophy did not play a significant role in the enhancement of pulmonary functions after septoplasty.

Conclusions: A favorable outcome in pulmonary function was observed in patients with deviated nasal septum after septoplasty. However, due to limited sample size, it is advisable to conduct the study in a larger sample to validate these results.

Keywords: Deviated nasal septum, Pulmonary function tests, Septoplasty, Septorhinoplasty

\section{INTRODUCTION}

The nasal septum divides the nasal cavity into two halves consists of cartilaginous and osseous tissues. ${ }^{1}$ The deviated nasal septum, involving a dislocation of the nasal septum, is mostly confined to the septal bone, septal cartilage and sometimes both. ${ }^{2}$ It is estimated that up to $90 \%$ of individuals have nasal septal deformities without any symptoms. ${ }^{1,3}$ Trauma along with genetic and environmental factors are the main common etiologic factors for nasal septal deviation. ${ }^{2}$
The nose is the most physiologic route of respiration performs important functions such as filtration, heat exchange, humidification, and olfaction. ${ }^{4}$ Nasal septum deviations and the consequent pathological change of the nasal turbinates are frequently identified to cause nasal handicap to the patients. ${ }^{5}$ These deviations may lead to one-sided obstruction and can change the resonance of vocalization along with respiratory and aesthetic problems. ${ }^{1}$ 
Nasal breathing augments the capability of the respiratory system to warm and humidify the inspired air. Deviation of the nasal septum and consequent pathological alterations are frequently identified to cause changes in respiratory mechanics and arterial blood compositions. Deviation of normal pulmonary functions leads to dysfunction of the respiratory system and this affects the functions and vitality of other related systems., ${ }^{4,6}$ Pulmonary function tests provide useful information on the state of the airways, lung volumes and lung function. A clinically significant deviated septum is defined as one with sufficient deviation that would make the patient a candidate for septoplasty if the nasal obstructive symptoms do not respond to medical therapy. ${ }^{8}$ Theoretically, surgical correction of the deviated septum by septoplasty should improve nasal breathing as well as pulmonary mechanics.

Previous studies conducted demonstrated that nasal obstruction had a deleterious effect on pulmonary function. ${ }^{6}$ Furthermore, it was observed that the degree of symptomatic improvement succeeding corrective nasal surgery could be expected by preoperative pulmonary function testing. ${ }^{7}$ Hence, deviated nasal septum poses variable resistance to inspired air currents, which directly and indirectly influences the efficiency of pulmonary functions. Only one study has been done so far in the literature regarding the effectiveness of septoplasty on pulmonary function. This is the first study done in India in this regard. Hence this prompted us to examine the effect on pulmonary function in symptomatic deviated nasal septum cases following septoplasty.

\section{METHODS}

This was a 1-year observational study conducted at the Department of Otorhinolaryngology and Head and Neck Surgery from January 2013 to January 2014. Prior to the commencement, the study was granted by the Institutional Ethical and Research Committee. Patients fulfilling the selection criteria were identified and explained about the nature of the study and a written and informed consent was took before enrollment. Systemic random sampling was carried out where every fourth patient with symptomatic deviated nasal septum was incorporated in the study. A total of 35 patients were comprised in the study. Among them, 31 patients undertook septoplasty while 4 patients undertook septorhinoplasty.

\section{Selection criteria}

Patients with symptomatic deviated nasal septum with no previous pulmonary disorders and who were willing to undergo septoplasty were comprised in the study. All cases with known restrictive or obstructive pulmonary diseases, nasal polyps and space-occupying lesions of the nose and paranasal sinuses, and allergic and vasomotor rhinitis were exempted from the study.

\section{Data collection}

Demographic data including age, sex, and complaints of nasal obstruction, the side and duration of nasal obstruction, headache, sneezing, previous history of trauma and prior surgery were enquired from the patient. Physical examination including anterior and posterior rhinoscopy was performed. These findings were recorded on a predesigned and pretested proforma.

Diagnostic nasal endoscopy using 4-mm and 0-degree John Hopkins rigid nasal endoscopy was performed to validate the clinical diagnosis. The laterality of deviation and the involvement of the Cottle's areas, the type of deviation classified according to Cottle's classification, the involvement of bony/cartilaginous septum, and the presence of other contributory factors such as spur and inferior turbinate hypertrophy (ITH) were noted.

Pre- and postoperative pulmonary function tests were performed on the day before surgery and 6 weeks after septoplasty using RMS Helios 702 spirometer. Forced vital capacity (FVC), forced expiratory volume in 1 second (FEV1), peak expiratory flow rate (PEF), and the ratio of FEV1 to FVC were the parameters measured.

Septoplasty was performed under local anesthesia. Nasal packing was done post-surgery and removed on the first day after surgery. Patients were administered with parenteral antibiotics followed by oral antibiotics, antihistamines, and nonsteroidal anti-inflammatory drugs for 1 week after surgery. No complications were detected during postoperative follow-up periods.

\section{Data analysis}

The data obtained were coded and tabulated into Microsoft excel worksheet. SPSS v17.0 was used to analyze the data. The significance of difference between the means was assessed and comparison was done using ANOVA and paired and unpaired t test. A p-value $\leq 0.05$ was considered as statistically significant.

\section{RESULTS}

The demographic including clinical characteristics of the study participants are summarized in Table 1 . In the present study, most of the patients $(71 \%)$ were male patients with mean age of $28.5 \pm 6.41$ years while $29 \%$ were female with mean age of $23.9 \pm 5.38$ years. All the patients in this study (100\%) suffered from nasal block with a mean duration of $3.8 \pm 3.13$ years, $25.7 \%$ with headache, $20 \%$ with sneezing and $22.9 \%$ of the patients had history of trauma. The deviation was most commonly seen on the left side (54.28\%), affecting mostly the cartilaginous septum (54\%). Majority of the patients with deviated septum were observed with absence of spur (28\%), caudal dislocation (28.57\%) and ITH (40\%). According to Cottle's Classification, most of the patients $(66 \%)$ had obstruction. The most frequent deviation was seen in area $4(20 \%)$ followed by areas $2,4(17.14 \%)$. 
Table 1: Demographic and clinical characteristics of the study participants.

\begin{tabular}{|c|c|c|}
\hline Variables & & $\mathbf{N}(\%)$ \\
\hline \multirow{2}{*}{ Gender } & Male & $25(71)$ \\
\hline & Female & $10(29)$ \\
\hline \multirow{3}{*}{ Age distribution (yrs.) } & $18-20$ & $12(34.2)$ \\
\hline & $21-30$ & 16 (45.7) \\
\hline & $31-40$ & $7(20)$ \\
\hline \multirow{3}{*}{ Laterality of nose block } & Right & $10(28.57)$ \\
\hline & Left & $16(45.71)$ \\
\hline & Bilateral & $9(25.71)$ \\
\hline \multirow{6}{*}{ Duration of Nose Block (yrs.) } & $0-2$ & $15(42.8)$ \\
\hline & $2-4$ & $12(34.2)$ \\
\hline & $4-6$ & $4(11.42)$ \\
\hline & $6-8$ & 0 \\
\hline & $8-10$ & $4(11.42)$ \\
\hline & Above 10 & $1(2.8)$ \\
\hline \multirow{2}{*}{ Headache } & Presence & $9(25.27)$ \\
\hline & Absence & $26(74.7)$ \\
\hline \multirow{2}{*}{ Sneezing } & Presence & $7(20)$ \\
\hline & Absence & $28(80)$ \\
\hline \multirow{2}{*}{ Traumatic septal deviation } & Presence & $8(22.85)$ \\
\hline & Absence & $27(77.14)$ \\
\hline \multirow{3}{*}{ External appearance of nose } & Crooked nose & $6(17.14)$ \\
\hline & Hump & $1(2.8)$ \\
\hline & None & $28(80)$ \\
\hline \multirow{3}{*}{ Laterality of deviated septum } & Right & $11(31.4)$ \\
\hline & Left & $19(54.28)$ \\
\hline & S-shaped & $5(14.28)$ \\
\hline \multirow{3}{*}{ Bony and cartilaginous deviation } & Bony & $6(17.14)$ \\
\hline & Cartilaginous & $19(54.28)$ \\
\hline & Both & $10(28.57)$ \\
\hline \multirow{4}{*}{ Spur } & Right present & $3(8.5)$ \\
\hline & Left present & $3(8.5)$ \\
\hline & Bilateral present & $1(2.8)$ \\
\hline & Absence & $28(80)$ \\
\hline \multirow{3}{*}{ Caudal dislocation } & Right & $6(17.14)$ \\
\hline & Left & $4(11.42)$ \\
\hline & Absence & $25(28.57)$ \\
\hline \multirow{4}{*}{ Presence of ITH } & Right & $13(37.14)$ \\
\hline & Left & $4(11.42)$ \\
\hline & Bilateral & $4(11.42)$ \\
\hline & Absent & $14(40)$ \\
\hline \multirow{2}{*}{ Cottle's classification of DNS } & Obstruction & $23(65.71)$ \\
\hline & Impaction & $12(34.28)$ \\
\hline \multirow{6}{*}{ Cottle's areas involved } & Area 2 & $10(28.53)$ \\
\hline & Area 4 & $7(20)$ \\
\hline & Area 5 & $5(14.2)$ \\
\hline & Area 2,4 & $6(17.45)$ \\
\hline & Area 4,5 & $4(11.42)$ \\
\hline & Area $2,4,5$ & $3(8.5)$ \\
\hline
\end{tabular}

DNS: Deviated nasal septum; ITH: Inferior tubinate hypertrophy. 
Table 2: Demographic and clinical characteristics of the study participants in relation to pulmonary function tests.

\begin{tabular}{|c|c|c|c|c|c|}
\hline \multirow{2}{*}{ Findings } & & \multicolumn{2}{|c|}{ FVC (mean \pm SD $)$} & \multicolumn{2}{|c|}{ FEV1 (mean \pm SD) } \\
\hline & & Preoperative & Postoperative & Preoperative & Postoperative \\
\hline \multirow{3}{*}{ Age distribution (Yrs.) ${ }^{+}$} & $18-20$ & $69.17 \pm 14.93 *$ & $84.25 \pm 11.05 *$ & $71.75 \pm 19.89 *$ & $83.58 \pm 17.63^{*}$ \\
\hline & $21-30$ & $70.94 \pm 15.19 *$ & $79.8 \pm 14.51 *$ & $77.19 \pm 16.14 *$ & $84.94 \pm 15.74 *$ \\
\hline & $31-40$ & $74 \pm 20.34$ & $81.7 \pm 18.32$ & $79.71 \pm 14.64$ & $85.29 \pm 16.13$ \\
\hline \multirow{2}{*}{ Sex distribution ${ }^{\dagger}$} & Male & $73.04 \pm 16.45^{*}$ & $83.8 \pm 13.3^{*}$ & $76.04 \pm 18.24 *$ & $84.68 \pm 16.28^{*}$ \\
\hline & Female & $65.70 \pm 13.42 *$ & $76.8 \pm 15.1 *$ & $75.3 \pm 14.50^{*}$ & $83.7 \pm 16.23 *$ \\
\hline \multirow{3}{*}{ Laterality of nose block ${ }^{+}$} & Right & $66 \pm 14.68 *$ & $75.4 \pm 14.89 *$ & $72.2 \pm 15.92 *$ & $79.20 \pm 17.14^{*}$ \\
\hline & Left & $74.25 \pm 15.34 *$ & $84.56 \pm 12.25 *$ & $81.75 \pm 15.05 *$ & $89.9 \pm 10.52 *$ \\
\hline & Bilateral & $70.56 \pm 18.05^{*}$ & $84 \pm 15.10^{*}$ & $69.33 \pm 19.78 *$ & $80.33 \pm 20.97 *$ \\
\hline \multirow{4}{*}{$\underset{+}{\text { Duration of Nose Block (Yrs.) }}$} & $0-2$ & $67 \pm 13.8^{*}$ & $80.4 \pm 13.73 *$ & $77.07 \pm 16.94 *$ & $86.8 \pm 14.01 *$ \\
\hline & $2-4$ & $77.42 \pm 18.92 *$ & $84.5 \pm 16.43^{*}$ & $73.58 \pm 18.74 *$ & $80.08 \pm 19.07^{*}$ \\
\hline & $4-6$ & $67.6 \pm 11.32 *$ & $83 \pm 11.04^{*}$ & $75.4 \pm 15.58$ & $87.6 \pm 15.09$ \\
\hline & $>6$ & $72.67 \pm 15.04$ & $77.67 \pm 10.78$ & $79 \pm 21.79 *$ & $80.67 \pm 22.14 *$ \\
\hline \multirow{2}{*}{ Headache $^{\dagger}$} & Absent & $71.88 \pm 16.19 *$ & $82.00 \pm 13.31 *$ & $75.23 \pm 17.88^{*}$ & $83.03 \pm 15.54^{*}$ \\
\hline & Present & $68.22 \pm 15.22 *$ & $81.22 \pm 16.63 *$ & $77.55 \pm 15.22 *$ & $88.33 \pm 17.69 *$ \\
\hline \multirow{2}{*}{ Sneezing $^{+}$} & Absent & $72.07 \pm 16.25^{*}$ & $83.85 \pm 13.53^{*}$ & $78.28 \pm 16.02 *$ & $87.28 \pm 14.37 *$ \\
\hline & Present & $66.42 \pm 14.08 *$ & $73.57 \pm 13.61^{*}$ & $66.00 \pm 18.72 *$ & $72.85 \pm 18.22 *$ \\
\hline \multirow{2}{*}{ Traumatic septal deviation ${ }^{\dagger}$} & Absent & $75.46 \pm 19.44 *$ & $82.61 \pm 18.56^{*}$ & $73.43 \pm 16.66^{*}$ & $84.68 \pm 14.56^{*}$ \\
\hline & Present & $72.57 \pm 15.29 *$ & $86.43 \pm 14.24 *$ & $65.57 \pm 11.74 *$ & $80.29 \pm 11.28 *$ \\
\hline \multirow{2}{*}{ Crooked Nose $^{+}$} & Absent & $75.46 \pm 19.44 *$ & $82.61 \pm 18.56^{*}$ & $73.43 \pm 16.6^{*}$ & $84.68 \pm 14.56^{*}$ \\
\hline & Present & $72.57 \pm 15.29 *$ & $86.43 \pm 14.24 *$ & $65.57 \pm 11.74 *$ & $80.29 \pm 11.28 *$ \\
\hline \multirow{2}{*}{ Hump $^{+}$} & Absent & $75.46 \pm 19.44 *$ & $82.61 \pm 18.56^{*}$ & $73.43 \pm 16.66^{*}$ & $84.68 \pm 14.56^{*}$ \\
\hline & Present & $72.57 \pm 15.29 *$ & $86.43 \pm 14.24 *$ & $65.57 \pm 11.74 *$ & $80.29 \pm 11.28 *$ \\
\hline \multirow{3}{*}{$\begin{array}{l}\text { Laterality of deviated } \\
\text { septum }^{+}\end{array}$} & Right & $65.27 \pm 14.14$ & $76.64 \pm 14.71$ & $71.55 \pm 15.26^{*}$ & $79.27 \pm 16.26^{*}$ \\
\hline & Left & $73.74 \pm 15.10 *$ & $85.21 \pm 11.96^{*}$ & $81.05 \pm 15.20 *$ & $91.16 \pm 10.79 *$ \\
\hline & S-shaped & $72.8 \pm 21.67$ & $80.2 \pm 18.58$ & $65.4 \pm 23.10$ & $70 \pm 21.21$ \\
\hline \multirow{3}{*}{$\begin{array}{l}\text { Bony and cartilaginous } \\
\text { deviation }{ }^{+}\end{array}$} & Bony & $73.50 \pm 18.50$ & $85.17 \pm 14.70$ & $81.83 \pm 17.40$ & $91 \pm 10.65$ \\
\hline & Cartilaginous & $72.84 \pm 16.63 *$ & $81.89 \pm 13.8 *$ & $74.58 \pm 18.66^{*}$ & $82.63 \pm 17.45^{*}$ \\
\hline & Both & $56 \pm 19.47 *$ & $62.74 \pm 21.07 *$ & $74.6 \pm 14.3 *$ & $83.8 \pm 16.20 *$ \\
\hline \multirow{4}{*}{ Spur $^{+}$} & Absent & $73.07 \pm 18.77 *$ & $80.4 \pm 18.44^{*}$ & $73.29 \pm 16.78$ & $83.86 \pm 14.01$ \\
\hline & Right & $73 \pm 19.15$ & $77 \pm 20.78$ & $66.13 \pm 12.89$ & $71.67 \pm 17.55$ \\
\hline & Left & $88.67 \pm 15.88$ & $88.63 \pm 10.40$ & $77 \pm 12.12$ & $88.33 \pm 9.71$ \\
\hline & Bilateral & 90 & 95 & 76 & 78 \\
\hline \multirow{3}{*}{ Caudal dislocation $^{+}$} & Absence & $69.28 \pm 14.75^{*}$ & $78.80 \pm 13.16^{*}$ & $75.04 \pm 18.35 *$ & $82.80 \pm 16.44 *$ \\
\hline & Right & $82.66 \pm 7.17$ & $93.66 \pm 8.40$ & $85.16 \pm 11.46$ & $94.66 \pm 7.20$ \\
\hline & Left & $63.75 \pm 15.30$ & $82.75 \pm 18.83$ & $66.75 \pm 9.60 *$ & $79.00 \pm 19.76^{*}$ \\
\hline \multirow{4}{*}{ Presence of ITH $^{\dagger}$} & Absent & $72.50 \pm 15.99 *$ & $85.50 \pm 11.56^{*}$ & $73.28 \pm 16.88 *$ & $82.07 \pm 16.64 *$ \\
\hline & Right & $71.38 \pm 15.05^{*}$ & $81.53 \pm 15.19 *$ & $79.53 \pm 18.06 *$ & $89.92 \pm 15.80 *$ \\
\hline & Left & $70.00 \pm 13.88^{*}$ & $73.00 \pm 11.51^{*}$ & $78.75 \pm 17.72 *$ & $82.75 \pm 16.02 *$ \\
\hline & Bilateral & $65.0 \pm 23.79$ & $78.50 \pm 19.97$ & $69.75 \pm 17.03$ & $76.25 \pm 14.36$ \\
\hline \multirow{2}{*}{ Cottles classification of DNS ${ }^{\dagger}$} & Obstruction & $73.35 \pm 16.33 *$ & $83.48 \pm 13.6^{*}$ & $76.48 \pm 18.78^{*}$ & $85.26 \pm 17.18 *$ \\
\hline & Impaction & $66.3 \pm 14.27 *$ & $78.58 \pm 14.7 *$ & $74.58 \pm 13.8^{*}$ & $82.75 \pm 14.11 *$ \\
\hline \multirow{2}{*}{$\begin{array}{l}\text { valve area vs. other Cottle } \\
\text { areas }^{t}\end{array}$} & $\begin{array}{l}\text { Area to } \\
\text { involvement }\end{array}$ & $65.33 \pm 14.44 *$ & $76.41 \pm 15.07 *$ & $68.91 \pm 9.42 *$ & $76.83 \pm 12.59 *$ \\
\hline & Other area & $72.72 \pm 15.39 *$ & $83.95 \pm 12.73 *$ & $79.09 \pm 19.49 *$ & $88.27 \pm 16.82 *$ \\
\hline \multirow{2}{*}{$\begin{array}{l}\text { Septoplasty vs. } \\
\text { septorhinoplasty }\end{array}$} & Septoplasty & $72.19 \pm 16.26^{*}$ & $81.58 \pm 14.66^{*}$ & $76.4 \pm 17.79 *$ & $85.03 \pm 16.83^{*}$ \\
\hline & Rhinoplasty & $61.25 \pm 6.89$ & $83.5 \pm 7.85$ & $68.75 \pm 7.41$ & $79.5 \pm 6.57$ \\
\hline
\end{tabular}

DNS: Deviated nasal septum; *(paired t-test) Significant; † (unpaired t-test) not significant; ITH: Inferior tubinate hypertrophy; FVC: Forced vital capacity; FEV: Forced expiratory volume. 
Table 3: Demographic and clinical characteristics of the study participants in relation to pulmonary function tests.

\begin{tabular}{|c|c|c|c|c|c|}
\hline \multirow{2}{*}{ Findings } & & \multicolumn{2}{|c|}{ FEV1/FVC (mean \pm SD) } & \multicolumn{2}{|c|}{ PEFR (mean \pm SD) } \\
\hline & & Preoperative & Postoperative & Preoperative & Postoperative \\
\hline \multirow{3}{*}{ Age distribution (Years) ${ }^{\dagger}$} & $18-20$ & $105.33 \pm 23.28$ & $97.5 \pm 20.01$ & $55.17 \pm 12.91 *$ & $64.92 \pm 15.73^{*}$ \\
\hline & $21-30$ & $109.69 \pm 12.94$ & $100.75 \pm 14.04$ & $60.44 \pm 20.51 *$ & $64.38 \pm 21.12 *$ \\
\hline & $31-40$ & $109.71 \pm 14.05$ & $99.71 \pm 10.25$ & $55.43 \pm 20.02 *$ & $59.71 \pm 20.81 *$ \\
\hline \multirow{2}{*}{ Sex distribution ${ }^{+}$} & Male & $105.68 \pm 19.08$ & $99.32 \pm 16.85$ & $54.32 \pm 18.45^{*}$ & $60.4 \pm 20.41^{*}$ \\
\hline & Female & $114.5 \pm 7.54^{*}$ & $98.9 \pm 11.59 *$ & $65.9 \pm 13.56^{*}$ & $72 \pm 11.80^{*}$ \\
\hline \multirow{3}{*}{ Laterality of nose block ${ }^{\dagger}$} & Right & $109.9 \pm 13.73$ & $99.6 \pm 13.85$ & $58.2 \pm 15.34 *$ & $64.3 \pm 15.42 *$ \\
\hline & Left & $110.75 \pm 10.60 *$ & $100.13 \pm 11.86$ & $62 \pm 18.85^{*}$ & $68.38 \pm 21.91 *$ \\
\hline & Bilateral & $101.78 \pm 27.20$ & $97.11 \pm 22.72$ & $49.22 \pm 17.28$ & $54.78 \pm 14.99$ \\
\hline \multirow{4}{*}{$\begin{array}{l}\text { Duration of Nose Block } \\
\text { (Years) }\end{array}$} & $0-2$ & $114.53 \pm 5.76^{*}$ & $99.27 \pm 11.24 *$ & $63.8 \pm 14.11^{*}$ & $69.47 \pm 14.23 *$ \\
\hline & $2-4$ & $98 \pm 25.24$ & $96 \pm 21.56$ & $44.58 \pm 15.56^{*}$ & $49.92 \pm 16.18^{*}$ \\
\hline & $4-6$ & $111.2 \pm 9.57$ & $99.8 \pm 9.25$ & $70.2 \pm 11.32$ & $80.6 \pm 11.45$ \\
\hline & $>6$ & $107.67 \pm 9.07$ & $102.67 \pm 13.42$ & $62.33 \pm 26.95$ & $63.67 \pm 30$ \\
\hline \multirow{2}{*}{ Headache $^{+}$} & Absent & $106.19 \pm 18.98 *$ & $99.69 \pm 16.93^{*}$ & $58.11 \pm 20.28 *$ & $63.23 \pm 21.65^{*}$ \\
\hline & Present & $114.00 \pm 7.01$ & $97.77 \pm 10.18$ & $56.22 \pm 7.69 *$ & $65.11 \pm 7.67 *$ \\
\hline \multirow{2}{*}{ Sneezing $^{\dagger}$} & Absent & $109.35 \pm 11.49 *$ & $101.39 \pm 11.14^{*}$ & $58.42 \pm 17.63 *$ & $64.03 \pm 19.45^{*}$ \\
\hline & Present & $103.57 \pm 31.76$ & $90.42 \pm 25.81$ & $54.42 \pm 19.60 *$ & $62.42 \pm 18.15^{*}$ \\
\hline \multirow{2}{*}{ Traumatic septal deviation $^{\dagger}$} & Absent & $104.93 \pm 23.24 *$ & $96.18 \pm 18.97 *$ & $56.14 \pm 19.74 *$ & $62.89 \pm 19.30 *$ \\
\hline & Present & $110.29 \pm 6.34$ & $100.57 \pm 12.84$ & $58.57 \pm 16.36$ & $62.71 \pm 22.18$ \\
\hline \multirow{2}{*}{ Crooked Nose $^{\dagger}$} & Absent & $104.93 \pm 23.24 *$ & $96.18 \pm 18.97 *$ & $56.14 \pm 19.74 *$ & $62.89 \pm 19.30 *$ \\
\hline & Present & $110.29 \pm 6.34$ & $100.57 \pm 12.84$ & $58.57 \pm 16.36$ & $62.71 \pm 22.18$ \\
\hline \multirow{2}{*}{ Hump ${ }^{+}$} & Absent & $104.93 \pm 23.24 *$ & $96.18 \pm 18.97 *$ & $56.14 \pm 19.74 *$ & $62.89 \pm 19.30 *$ \\
\hline & Present & $110.29 \pm 6.34$ & $100.57 \pm 12.84$ & $58.57 \pm 16.36$ & $62.71 \pm 22.18$ \\
\hline \multirow{3}{*}{$\begin{array}{l}\text { Laterality of deviated } \\
\text { septum }^{+}\end{array}$} & Right & $110.09 \pm 13.05$ & $98.73 \pm 13.46$ & $59 \pm 14.79$ & $63 \pm 15.25$ \\
\hline & Left & $110.42 \pm 9.72 *$ & $101.68 \pm 11.46^{*}$ & $60.37 \pm 17.65 *$ & $67.63 \pm 20.10 *$ \\
\hline & S-shaped & $95.6 \pm 37.01$ & $90.8 \pm 29.04$ & $44.20 \pm 21.93$ & $50.4 \pm 18.929$ \\
\hline \multirow{3}{*}{$\begin{array}{l}\text { Bony and cartilaginous } \\
\text { deviation }{ }^{+}\end{array}$} & Bony & $112.17 \pm 11.92$ & $96.83 \pm 111.85$ & $53.83 \pm 13.40^{*}$ & $60.83 \pm 17.15^{*}$ \\
\hline & Cartilaginous & $104.11 \pm 20.9$ & $100.21 \pm 18.62$ & $56 \pm 19.47 *$ & $62.74 \pm 21.07 *$ \\
\hline & Both & $113.6 \pm 7.69 *$ & $98.70 \pm 10.67 *$ & $63 \pm 17.12$ & $67.3 \pm 16.85$ \\
\hline \multirow{4}{*}{ Spur ${ }^{+}$} & Absent & $103.29 \pm 22.13$ & $96.79 \pm 19.54$ & $55.46 \pm 19.54$ & $61.61 \pm 20.04$ \\
\hline & Right & $119 \pm 18.24$ & $100 \pm 17.51$ & $50 \pm 17.08$ & $53.33 \pm 15.27$ \\
\hline & Left & $114.67 \pm 2.08$ & $101.67 \pm 9.07$ & $74 \pm 9.64$ & $83.3 \pm 7.63$ \\
\hline & Bilateral & 118 & 82 & 57 & 65 \\
\hline \multirow{3}{*}{ Caudal dislocation $^{+}$} & Absence & $109.16 \pm 17.54 *$ & $99.16 \pm 16.35^{*}$ & $58.92 \pm 17.69 *$ & $66.36 \pm 18.32 *$ \\
\hline & Right & $85.16 \pm 11.46$ & $94.66 \pm 7.20$ & $66.75 \pm 9.60 *$ & $79.00 \pm 19.76^{*}$ \\
\hline & Left & $107.25 \pm 21.68$ & $104.75 \pm 17.68$ & $53.00 \pm 18.83$ & $54.00 \pm 16.95$ \\
\hline \multirow{4}{*}{ Presence of ITH $^{\dagger}$} & Absent & $103.92 \pm 24.96$ & $98.42 \pm 19.47$ & $47.92 \pm 15.76^{*}$ & $54.35 \pm 18.39 *$ \\
\hline & Right & $111.00 \pm 6.28$ & $100.84 \pm 12.62$ & $68.53 \pm 4.17 *$ & $77.07 \pm 12.05^{*}$ \\
\hline & Left & $112.25 \pm 8.18$ & $96.75 \pm 14.38$ & $66.00 \pm 15.23 *$ & $68.00 \pm 16.71 *$ \\
\hline & Bilateral & $110.00 \pm 14.14$ & $99.00 \pm 12.83$ & $47.75 \pm 17.44$ & $48.75 \pm 5.47$ \\
\hline \multirow{2}{*}{ Cottles classification of DNS ${ }^{+}$} & Obstruction & $105.65 \pm 19.26$ & $98.96 \pm 17.33$ & $59.09 \pm 20.01 *$ & $65.4 \pm 20.828^{*}$ \\
\hline & Impaction & $113.08 \pm 10.5^{*}$ & $99.67 \pm 11.33^{*}$ & $54.83 \pm 12.90 *$ & $60.4 \pm 14.99 *$ \\
\hline \multirow{2}{*}{$\begin{array}{l}\text { valve area vs. other Cottle } \\
\text { areas }^{t}\end{array}$} & $\begin{array}{l}\text { Area to } \\
\text { involvement }\end{array}$ & $107.41 \pm 14.42$ & $102.83 \pm 13.32$ & $51.16 \pm 15.34 *$ & $53.75 \pm 16.31 *$ \\
\hline & Other area & $109.59 \pm 18.28 *$ & $96.72 \pm 16.42 *$ & $62.18 \pm 17.89 *$ & $70.45 \pm 17.26^{*}$ \\
\hline \multirow{2}{*}{$\begin{array}{l}\text { Septoplasty vs. } \\
\text { Septorhinoplasty }\end{array}$} & Septoplasty & $107.68 \pm 17.99 *$ & $99.68 \pm 16.25^{*}$ & $57.90 \pm 18.25^{*}$ & $64.97 \pm 18.94 *$ \\
\hline & Rhinoplasty & $112.25 \pm 2.5$ & $95.5 \pm 3.69$ & $54 \pm 18.45$ & $55.5 \pm 16.03$ \\
\hline
\end{tabular}

DNS: Deviated nasal septum; * (paired t-test) Significant; $†$ (unpaired t-test) not significant; ITH: Inferior tubinate hypertrophy; FVC: Forced vital capacity; FEV: Forced expiratory volume; PEFR: peak expiratory flow rate. 
Table 4: Pre and postoperative pulmonary function test results in patients with nasal septal deviation.

\begin{tabular}{|llll|}
\hline Variables & Preoperative $($ mean \pm SD) & Postoperative $($ mean \pm SD) & P value \\
\hline FEV1\% & $75.8 \pm 17.05$ & $84.4 \pm 16.03$ & $<0.001^{*}$ \\
\hline FVC\% & $70.9 \pm 15.81$ & $81.8 \pm 13.98$ & $<0.001^{*}$ \\
\hline PEFR\% & $57.6 \pm 17.8$ & $63.7 \pm 7.53$ & $0.005^{*}$ \\
\hline FEV1/FVC\% & $108.2 \pm 16.98$ & $99.2 \pm 15.36$ & $<0.001^{*}$ \\
\hline
\end{tabular}

*Significant; FVC: Forced vital capacity; FEV: Forced expiratory volume; PEFR: peak expiratory flow.

The pre and post-demographic and clinical characteristics of the study participants in relation to pulmonary function tests are given in Tables 2 and 3. The postoperative values of FEV1\% $(\mathrm{p} \leq 0.001), \mathrm{FVC} \%(\mathrm{p} \leq 0.001), \mathrm{PEF} \%$ $(p=0.005)$ and FEV1/FVC $\%(p \leq 0.001)$ were higher than the preoperative ones, and the results were statistically significant. Clinically significant improvement in the pulmonary functions was noted among males in the 2130-year age group, with left-sided nose block, preceding trauma, hump, left-sided deviation, and the cartilaginous part of the septum. However, age, gender, laterality and duration of deviation, headache, sneezing, part of septum involved, spur, caudal location, and ITH did not play a role in the enhancement of pulmonary functions after septoplasty.

Majority of the patients underwent septoplasty (88.57\%) compared to septorhinoplasty $(11.42 \%)$. The mean postoperative values of $\mathrm{FVC} \% \quad(\mathrm{p}=0.000), \mathrm{FEV} 1 \%$ $(\mathrm{p}=0.000)$ and PEFR $(\mathrm{p}=0.000)$ were higher than the preoperative values and these results were statistically significant. Also, patients with septorhinoplasty, the postoperative values of FVC\% $(\mathrm{p}=0.406)$, FEV1\% $(p=0.660)$, FEV1/FVC $(p=0.91)$ and PEFR $(p=0.200)$ were higher than the preoperative values but these results were not statistically significant. The mean improvement is more in cases with septoplasty. Though this is clinically significant, it is not statistically significant, calculated using unpaired t-test, FVC ( $\mathrm{p}=0.141)$, FEV1 $(\mathrm{p}=0.444)$, FEV1/FVC $(\mathrm{p}=0.079)$ and PEFR $(\mathrm{p}=0.197)$. The mean pre- and postoperative pulmonary function test results in patients with nasal septal deviation is shown in Table 4. The results obtained statistically significant in both the groups.

\section{DISCUSSION}

Difficulty in nasal breathing is perhaps the most common grievance in rhinologic practice. Among the main causes, nasal septum deviation accounts first. ${ }^{9}$ Studies have quoted that $60-90 \%$ of the population have a deviated septum. ${ }^{10,11}$ Studies conducted by Podoshin et al showed varying prevalence rates ranging from $0.93 \%$ in India to $55 \%$ in Greece. ${ }^{12}$

Most patients in the present study were in the age-group of 21-30 years with striking male preponderance. According to a study performed by Amer and Abdullah, the frequency of septal deviation was found to be $65 \%$ in men and $35 \%$ in women; it was also found that the age incidence of most patients is between second and fifth decades. Other studies in the literature also reported the same. ${ }^{13-15}$ This may be due to higher chances of trauma and environmental exposure in men. ${ }^{16}$ Also, in all agegroups, improvement in the pulmonary functions was observed after septoplasty. However, in the age-group of 31-40 years, the mean improvement was lesser in pulmonary function tests as compared with the other agegroups. This could suggest that, as the age increases, there is a lesser improvement in the pulmonary functions even after septoplasty.

Left-sided nasal obstruction/block was the most common complaint followed by headache, trauma and sneezing. This was comparable to the other studies in the literature. ${ }^{13,14,16,17}$ Also, a significant improvement in FVC and FEV1 was observed in all the cases, while the PEFR and FEV1/FVC were significant in cases of right-sided nose block and left-sided nose block only. In left-sided nose block, the mean improvement in FVC and FEV 1 was greater when compared to right-sided nose block and showed better improvement. Also, the mean improvement in FVC and FEV1 was found to be more among patients without sneezing, head ache and traumatic septal deviation than that of patients with symptoms of sneezing, head ache and trauma. In addition, our study showed that patients with nose block of a shorter duration (0-4 years) showed better improvement in pulmonary functions after septoplasty. This suggests that with an increased duration of nose block or kind of respiratory disorder, the mean improvement in the pulmonary function decreases. ${ }^{6,18}$ With regard to external appearance of the nose, none of the patients reported any external deformity followed by crooked nose and hump. Among the latter two cases, the post-operative values of patients with and without crooked nose and hump were higher than the preoperative values. However, no studies are available to support these findings.

In our study, most of the patients reported deviated septum to the left followed by right and S-shaped. However, other studies have shown right-sided deviated septum to be more common. ${ }^{19}$ It depends on the side of the trauma inflicted, the birth position of the patient and other developmental, genetic as well as environmental factors. Also, left-sided deviation showed better improvement in pulmonary function. However, there are no other studies indicating whether the side of the deviated septum plays a role in the improvement of pulmonary functions after septoplasty. 
The site of deviation as well as degree were important to assess the nasal septal deviation. In our study, cartilaginous septum was the common deviation found followed by the bony septum and both the parts. ${ }^{20}$ Though the mean post-operative values were higher than pre-operative values, correction of cartilaginous deviation showed more improvement in the pulmonary functions as compared to cases with bony deviation. This could be due to the better accessibility of the cartilaginous septum and hence better correction leading to improved functioning. There are no studies investigating the role of bony and cartilaginous septum in the improvement of pulmonary functions.

Even though the mean post-operative values were higher than the pre-operative values in all the patients, the difference in the pulmonary functions between the groups in all the above variables, were not statistically or clinically significant. Hence, all the above variables (age, gender, laterality and duration of deviation, headache, sneezing, part of septum involved, spur, caudal location, ITH) did not play a role in the enhancement of pulmonary functions after septoplasty. However, there is a paucity of data to validate these results.

In our study, Cottle's classification was adapted to assess the degree of nasal septal deviation. Impaction and obstruction were the two conditions encountered by patients in our study. Both cases of obstruction and impaction showed improvement in pulmonary function after septoplasty, with statistically significant results. However, the difference in the pulmonary functions between the groups with obstruction and impaction was not statistically significant. Furthermore, the deviation was further classified based on Cottle's areas. The most frequent deviation was seen in area 4 followed by area 5 . The deviation was also seen in areas 2 and 4 combined followed by area 4 and 5 and area 2, 4, and 5. In contrast, a study by Rehman et al reported area $1(19 \%)$ had the most frequent septal deviation followed by area 3 $(10.48 \%)$ and area $4(7 \%) .^{21}$ The deviation was also seen in combinations of areas 2, 3 and $4(21.44 \%)$ followed by area 2 and $3(20.04 \%)$.

In our study, $88.57 \%$ of the patients underwent septoplasty while $11.42 \%$ underwent septorhinoplasty. In both the two groups, the postoperative values of pulmonary functions were higher than the preoperative ones, but the results were statistically significant in septoplasty group. This suggests that septoplasty leads to a favorable outcome in pulmonary function tests. However, the difference in the pulmonary functions between the groups were not statistically significant. There are only four patients who underwent septorhinoplasty. Hence, this could be the cause for the lesser improvement in pulmonary functions as compared to septoplasty. Likewise, a study conducted by Bulcan et al in Turkey also reported statistically significant improvement in both nasal symptoms and pulmonary function test values after surgical treatment for nasal septal deviation. They also observed that nine patients had bronchial hyper-responsiveness before surgery and after surgery three of them showed negative bronchial provocation test and six showed a decrease in the rate of bronchial hyper-responsiveness. This suggested that septoplasty may have favorable effects on BHR. ${ }^{2}$ However, as per our understanding, there has been no study corroborating the effectiveness of septorhinoplasty in the improvement of pulmonary functions. So far, only two studies have been conducted till date to evaluate the effectiveness of septoplasty on pulmonary functions. Ours is the only study done in this issue in India., ${ }^{22}$

Our study showed that septoplasty leads to a favorable outcome on nasal obstruction as well as pulmonary functions, corroborated and endorsed by the improvement in the pulmonary function tests. Septorhinoplasty leads to clinical improvement, but the improvement in pulmonary function is not statistically significant which may be accounted for a small sample size. However, there is a need for large multicentric studies to validate the results.

\section{ACKNOWLEDGMENTS}

Both the authors have contributed equally in the development of manuscript.

Funding: No funding sources

Conflict of interest: None declared

Ethical approval: The study was approved by the Institutional Ethics Committee

\section{REFERENCES}

1. Buyukertan M, Keklikoglu N, Kokten G. A morphometric consideration of nasal septal deviations by people with paranasal complaints; A computed tomography study. Rhinol. 2003;41(1):21-4.

2. Bulcun E, Kazkayasi M, Ekici MA, Tahran FD, Ekici M. Effects of septoplasty on pulmonary function tests in patients with nasal septal deviation. J Otolaryngol Head Neck Surg. 2010;39(2):196202.

3. Moore K. Clinically oriented anatomy. USA: Williams and Wilkins; 1994.

4. Kurtaran H, Ark N, Sadikoglu F, Uğur KŞ, Yilmaz $\mathrm{T}$, Yildirim $\mathrm{Z}$, et al. The effect of anterior nasal packing with airway tubes on pulmonary function following septoplasty. Turk J Med Sci. 2009;39(4):537-40.

5. Baumann I, Baumann H. A new classification of septal deviations. Rhinol. 2007;45(3):220-3.

6. Ogura JH, Nelson JR, Dammkoehler R, Kawasaki M, Togawa K. Experimental Observations of the Relationships between Upper Airway Obstruction and Pulmonary Function. Ann Otol Rhinol Laryngol. 1964;73(2):381-403.

7. Ogura J, Unno T, Nelson J. Baseline values in pulmonary mechanics for physiologic surgery of the 
nose. Ann Otol Rhinol Laryngol. 1968;77(3):36797.

8. Ahmad R, Nicolas Y, Michael J, David A. Clinical assessment is an accurate predictor of which patients will need septoplasty. The Laryngoscope 2013;123(1):48-52.

9. Gray LP. Deviated nasal septum incidence and etiology. Ann Otol Rhinol Laryngol. 1978;87(3 suppl 2):3-20.

10. de Oliveira AKP, Elias E, Bettega SG, Mocellin M. Prevalence of deviated nasal septum in Curitiba, Brazil. Int Arch Otorhinolaryngol. 2005;9(4).

11. Soboczyński A, Skuratowicz A, Grzegorowski M. Nasal septum deviation in newborns. Acta otorhino-laryngologica Belgica. 1992;46(3):263-5.

12. Podoshin L, Gertner R, Fradis M, Berger A. Incidence and treatment of deviation of nasal septum in newborns. Ear Nose Throat J. 1991;70(8):485-7.

13. Amer S, Abdullah D, Ammar M. Assessment of nasal septal deformities by anterior rhinoscopy and nasal endoscopy. Tikrit Med J. 2010;16(1):14-20.

14. Teklal P, Prakash T. Comparative Study between Submucous Resection and Septoplasty to Manage Deviated Nasal Septum. Int J Recent Trends Sci Technol. 2014;9(3):318-20.

15. Ayaz R, Sajad H, Mushtaq A, Arsalan F. A Prospective Study of Nasal Septal Deformities in Kashmiri Population Attending a Tertiary Care Hospital. Int J Otorhinolaryngol Head Neck Surg. 2012;1(3):77-84.

16. Mahmood K, Fareed T, Tabassum R. Management of Deviated nasal septum. J Pharm Sci and Res. 2011;3(1):918-22.
17. Saad A, Ahmed K. Nasal Septal Deviation, pre and postoperative Symptoms Assessment. Med J Babylon. 2012;9(2):397-402.

18. Bergeron C, Tulic MK, Hamid Q. Airway remodelling in asthma: from benchside to clinical practice. Can Respir J. 2010;17(4):e85-93.

19. Stallman JS, Lobo JN, Som PM. The incidence of concha bullosa and its relationship to nasal septal deviation and paranasal sinus disease. Am J Neuroradiol. 2004;25(9):1613-8.

20. Yildirim I, Okur E. The prevalence of nasal septal deviation in children from Kahramanmaras, Turkey. Int J Pediatr Otorhinolaryngol. 2003;67(11):1203-6.

21. Rehman A, Hamid S, Ahmad M, Rashid AF. A Prospective Study of Nasal Septal Deformities in Kashmiri Population Attending a Tertiary Care Hospital. Int $\mathbf{J}$ Otorhinolaryngol Head Neck Surg. 2012;1(03):77.

22. van Egmond $M$, Rovers $M$, Hendriks $C$, van Heerbeek N. Effectiveness of septoplasty versus non-surgical management for nasal obstruction due to a deviated nasal septum in adults: study protocol for a randomized controlled trial. Trials. 2015;16(1):500.

Cite this article as: Panicker VB, Belaldavar BP. Effectiveness of septoplasty on pulmonary function tests in symptomatic deviated nasal septum cases: a prospective study. Int J Otorhinolaryngol Head Neck Surg 2018;4:800-7. 